\title{
Die „sekundären“ Geschlechtscharaktere der Insekten und das Problem der Vererbung des Geschlechts.
}

Von Dr. Otto Steche (Leipzig).

Auf der letzten Tagung der Deutschen Zoologischen Gesellschaft in Halle IgI2 habe ich über Untersuchungen berichtet, welche gemeinsam mit Herrn stud. KURT GEYER angestellt wurden und sexuelle Unterschiede in der Hämolymphe der Insekten betrafen. Wir konnten feststellen, daß durchgehend bei Lepidopteren und vereinzelt auch bei anderen Insektengruppen eine verschiedene Färbung der Lymphe auftritt in dem Sinne, daß sie bei den weiblichen Larven mehr oder weniger intensiv grün, seltener leuchtend gelb, bei den männlichen dagegen schwach gelblich oder farblos ist. Dieser Unterschied tritt nur bei phytophagen Larven auf (daher auch das verstreute Vorkommen außerhalb der Lepidopterengruppe) und lie $\beta$ sich durch spektroskopische Untersuchungen auf einen Gehalt des Weibchenblutes an Chlorophyllderivaten zurückführen, während das $0^{2}$ höchstens Xanthophyll oder gar keine derartigen Körper enthält. Das offenbar sehr wenig veränderte Chlorophyll ist im Blute in gelöstem Zustande vorhanden, da die Lymphe auch nach der Filtration durch Berkefeldkerzen, welche alle geformten Bestandteile zurückhalten, eine deutlich blaugrüne Farbe und das gleiche Spektrum wie die unveränderte Lymphe zeigt. Der sehr charakteristische, in Hunderten von Versuchen festgestellte Unterschied der beiden Geschlechter kann nun darauf beruhen, daß entweder das Männchenblut einen Stoff enthält, welcher das Chlorophyll zerstört oder daß es schon in den Darmzellen bei den $0^{t} 0^{*}$ abgebaut wird. Nach inzwischen angestellten Versuchen an Raupen von Lymantria monacha, die besonders geeignet ist, weil bei ihr keine Schwärzung der Hämolymphe durch Tyrosinase auftritt, dürfte die zweite Annahme zutreffen. Denn wenn man farblose Männchenlymphe in vitro zu der grünen Weibchenlymphe zusetzt, so 
tritt auch bei längerem Stehen keine Entfärbung ein. Eine Abhängigkeit dieser Erscheinung von den Geschlechtsdrüsen besteht nicht, denn Kastration und Transplantation, welche bei Lymantria dispar und monacha in zahlreichen Versuchsserien, zum Teil kombiniert mit Bluttransfusion ausgeführt wurden, ändern am normalen Verhalten absolut nichts.

Es erscheint danach die Annahme unabweislich, daß die bisher für sexuell völlig indifferent gehaltenen Darmzellen in ihren Stoffwechselreaktionen bei $\sigma^{*}$ und $\rho$ scharf geschieden sind. Die Farbe der Chlorophyllösung dient dafür bei den phytophagen Larven als ein sehr bequemer Jndikator. In der Annahme, daB wohl auch in den EiweiBkörpern der Lymphe ein sexueller Unterschied bestehen könnte, versuchten wir die Präzipitierung mit Serum, das durch Injektion von Kaninchen mit Hämolymphe gewonnen worden : war. Es ergab sich dabei aber kein Unterschied beim Zusammenbringen der Männchen. und Weibchenlymphe mit dem Serum. In beiden Fällen trat bei nicht zu großer Verdünnung ein deutlicher Ausfall ein. Das Resultat ist nicht verwunderlich, da die zweifellos zahlreich vorhandenen gleichen Eiweißkörper durch ihre Fällung die spezifische Reaktion der ungleichen verdecken werden. Der ganze Umweg erwies sich aber als unnötig, denn wir konnten feststellen, daß beim einfachen Zusammenbringen der Lymphe von $\sigma^{t}$ und $q$ der gleichen Art eine typische Reaktion, Schlierenbildung und Zusammenballung der Leukozyten eintritt, die sich nur quantitativ von der Reaktion bei der Mischung artfremder Lymphen (L. dispar und L. monacha) unterscheidet. Bringt man die Lymphen mehrerer Individuen des gleichen Geschlechts (z. B. 우 웅 von L. monacha) zusammen, so erfolgt keine Reaktion, sondern die Lymphe bleibt dauernd klar. Es verhalten sich also die beiden Geschlechter der gleichen Lepidopterenspezies chemisch zueinander wie fremde Arten (oder besser Rassen). Dadurch dürfte sich übrigens die von KOPEĆ 1 ) bei seinen Transfusionsversuchen ebenso wie von uns beobachtete Erscheinung erklären, daß die Raupen nach der Transfusion des geschlechtsfremden Blutes lange Zeit starr und bewegungslos verharren und anscheinend schwer geschädigt sind. Es tritt dann eben auch im Körper diese Reaktion zwischen den zwei Blutarten ein, die natürlich zu schweren mechanischen und chemischen Störungen Anlaß geben muß. Injektion eines Extraktes (resp. einer Suspension) von Chlorophyll in physiologischer Kochsalzlösung wurde

1) Kastration und Transplantation bei Schmetterlingen. Arch. f. Entw.-Mech. 33, 
von unseren Versuchstieren entschieden besser vertragen als Bluttransfusion, da hier die Störung durch die fremden Eiweißkörper des Blutes wegfiel.

In dieser Fällungsreaktion bei Mischung der Blutarten dürfte nun auch ein Mittel gegeben sein, die geschlechtliche Differenzierung des Stoffwechsels in den Fällen bei Insekten nachzuweisen, wo der Jndikator der Chlorophyllfarbe fehlt. In einigen Fällen haben wir bereits derartige Reaktionen erhalten (Coleopteren, Orthopteren). Der ausführliche Bericht über die gesamten Untersuchungen wird von Herrn GEYER an anderer Stelle erstattet werden, hier soll nur der u. E. theoretisch interessanteste Punkt der Ergebnisse etwas eingehender in seiner Bedeutung klargelegt werden. Es haben diese Untersuchungen in exakter Form den Beweis erbracht, daB ganze Zellterritorien des Insektenorganismus, in unserem Falle zum mindesten die Darmzellen, welche man bisher für sexuell indifferent hielt, tatsächlich geschlechtlich differenziert sind. Solche Unterschiede aber, speziell die spezifische Reaktion der Bluteiweißkörper, machen den SchluB fast unabweislich, da $B$ bei den Insekten der gesamte Organismus geschlechtlich differenziert ist. Es dürfte damit der Beweis geliefert sein für die zuletzt von KAMMERER in seiner schönen Ubersicht über den Ursprung der Geschlechtsunterschiede besonders energisch vertretene Auffassung, da B Geschlechtsunterschiede den Artunterschieden prinzipiell gleichzusetzen seien. Dadurch wird ein Verständnis der experimentellen Ergebnisse der Kastration und Transplantation bei Insekten (cf. MEISENHEIMER ${ }^{1}$ ) und KOPEĆ) sehr erleichtert. Denn wenn es keine Abänderungsmöglichkeit durch innere Sekretion oder Nerveneinfluß zwischen Geschlechtsdrüse und somatischen Merkmalen gibt, sondern alles „primäre" Sexualcharaktere sind, so ist die Erfolglosigkeit eines Eingriffs an den Geschlechtsorganen selbstverständlich. Ob KAMMERER im Recht ist, wenn er einen spezifischen Einfluß der inneren Sekretion der Keimdrüsen prinzipiell ausschaltet und dieser nur eine trophische Reizwirkung auf schon präformierte Anlagen zuschreibt, möchte ich bezweifeln. Es scheint mir, besonders im Hinblick auf die Kastrations- und Transplantationsergebnisse an Wirbeltieren ${ }^{2}$ ), richtiger, einen spezifischen EinfluB dieser inneren Sekretion anzunehmen, der sich nur je nach der Selbständigkeit der somatischen

1) Experimentelle Studien über Soma- und Geschlechtsdifferenzierung I u. II. Jena rgog u. IgIr.

2) Steinach, E.: Willkürliche Umwandlung von Säugetiermännchen in Tiere mit ausgeprägt weiblichen Charakteren etc. Pflügers Arch. 144. 
Merkmale verschieden stark geltend machen kann. Dafür spricht besonders die Korrelation der „primären“ und „sekundären“ Sexualcharaktere, die, wie alle derartige Korrelationen, wohl am leichtesten durch chemische oder nervöse Beziehungen der beteiligten Organe entstanden zu denken sind. Phylogenetisch lassen sich also primäre und sekundäre Sexualmerkmale denken, ontogenetisch haben wir aber bei den Insekten jedenfalls nur „primäre“ Sexualcharaktere vor uns, welche aller Wahrscheinlichkeit nach die spezifische Ausbildung aller somatischen Zellen bedingen. Der Begriff der primären und sekundären Séxualcharaktere ist also für die Insekten gegenstandslos.

Im Hinblick auf diese theoretische Konsequenz unserer Ergebnisse scheint mir nun eine Diskussion der äußerst interessanten Ergebnisse und Schlußfolgerungen am Platze zu sein, die GoLDSCHMIDT vor kurzem veröffentlicht hat (Erblichkeitsstudien an Schmetterlingen I. Untersuchungen über die Vererbung der sekundären Geschlechtscharaktere und des Geschlechts (Zeitschr. f. induktive Abstamm.- u. Vererbungslehre, Bd. VII, H. I. I9I2). GoLDSCHMIDT's Arbeit basiert auf eigenem Material und auf 8 Jahre hindurch fortgesetzten Zuchten eines Lepidopterologen, des Herrn BRAKE. Es handelt sich um Kreuzungen zwischen unserem Schwammspinner, Lymantria dispar und seiner japanischen Unterart, L. japonica. Bei diesen Zuchten traten nun unter bestimmten Bedingungen gynandromorphe Formen auf,

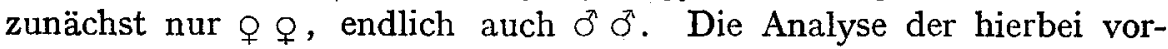
liegenden Vererbungsprobleme hat $G$. in sehr scharfsinniger Weise durchgeführt. Vom Standpunkt unserer Ergebnisse kann ich mich jedoch mit einem Teil seiner Schlußfolgerungen, nämlich vor allem der Rückführung der experimentellen Ergebnisse auf zytologische Verhältnisse nicht einverstanden erklären.

Die grundlegende Beobachtung GoldschmidT's ist die, daß bei bestimmten Kreuzungen, bei dispar $Q \times$ japonica ${ }^{t}$ in $\mathrm{F}_{1}$, bei der reziproken dispar $\delta^{3} \times$ japonica $ᄋ$ erst in $\mathrm{F}_{2}$, gynandromorphe $q$ ㅇ auftreten, die im Bau der Geschlechtsdrüsen anatomisch typische $q$ sind, in den übrigen Organen aber mehr oder weniger starke Anklänge an den Mänchenhabitus aufweisen. Um dies zu erklären, macht $G$. folgende Annahme: Er führt 2 Paare von Erbfaktoren ein: $F(Q)$ und $M\left(O^{*}\right)$ für „den weiblichen resp. männlichen Geschlechtsbestimmer"; G ( $Q$ ) und $\mathbf{A}\left(\sigma^{\pi}\right)$ für die sekundären Geschlechtsanlagen. $D a$ in den somatischen Charakteren Mischformen vorkommen, so muB jedes Geschlecht beide Faktoren, G und A, enthalten (das Ergebnis jeder Mischung wird dann durch Anderung der relativen Potenz dieser 
beiden Faktoren erklärt (s. u.). S. 28 macht nun G. für die sekundären Charaktere folgende Bemerkung: „Es dürften sich somit die Gesamtheit dieser Charaktere, die so ziemlich die meisten Organsysteme betreffen, auf Grundlage eines Erbfaktors vererben, resp. einer Anzahl streng korrelierter, was für die theoretische Betrachtung identisch ist.“ Dieser Punkt gibt Anlaß zu verschiedenen Einwänden. Wie G. selbst zugibt, ist die Zahl der diesem Faktor unterstehenden Merkmale tatsächlich sehr groB. G. selbst bespricht folgende Punkte:

Flügel: Färbung, Zeichnung, Form, Größe.

Fühler: Breite, Länge der Fiedern, Dornen der Fiedern, Haare und Schuppen am Schaft, Terminalborste.

Abdomen: Form, Größe, Behaarung, Afterwolle.

Genitalien: $0^{\top}$ : Chitinring, Saccus, Uncus, Valvae, Penis.

Q: Ostium bursae, laminae abdominales, 2 Paar Apophysen.

Schuppen: Form.

Instinkte: Flatterinstinkt, Kopulationstrieb.

Jeder einzelne dieser Punkte setzt sich nun wieder aus einer großen Anzahl von Einzelfaktoren zusammen, die selbständig abändern können (s. u.).

Dazu ließe sich noch eine große Anzahl anderer morphologischer Merkmale fügen, die schon bekannt war, nur von $G$. wegen ihrer geringeren Deutlichkeit nicht berücksichtigt ist. Es kommen nun aber auf Grund unsere Befunde wohl auch alle übrigen Organe hinzu, so daB dieser Erbfaktor G (resp. A) schließlich das gesamte Soma umfassen muß.

$\mathrm{Da} B$ diese Anlagen nun streng korrelativ vererbt werden, trifft entschieden nicht zu, wie aus G.'s eigenen Ausführungen vielfach hervorgeht. Es sind nämlich die Potenzschwankungen für die einzelnen Anlagen sehr verschieden. So sagt G. S. 3I, daB ,Flügelfärbung und äußere Genitalien zuerst nach dem anderen Geschlecht hin abändern". S. 30 steht: „Die Fühler wurden in diese Tabelle nicht aufgenommen, weil sie stärker als alle anderen Charaktere zur Männlichkeit tendieren."

Der beste Beweis dafür ist aber das Auftreten eines sehr feinen Mosaiks auf Flügeln, Fühlern und Abdomen. Dies läßt sich nur auf 2 Arten erklären: entweder so, daß die Faktoren für alle einzelnen Stellen (ev. die einzelnen Schuppen) unabhängig voneinander in ihrer Potenz bei der Vererbung variieren, oder, daß die zunächst einheitlich bestimmte Potenz sich'sekundär im Laufe der Ontogenese der einzelnen Teile ändert. Diese Schwankungen in der Potenz lassen 
sich jedenfalls mit dem Begriff der Korrelation, wie er bisher aufgefaßt wurde, nur schlecht vereinigen.

Wenn wir an der von G. postulierten Einteilung in Erbfaktoren für primäre und sekundäre Geschlechtsanlagen festhalten, so ergibt sich folgendes Verhältnis. Einesteils haben wir die Faktoren G(A) für die „sekundären Sexualcharaktere“. Diesen untersteht das gesamte Soma. Der Einfluß der „Geschlechtsbestimmer" $F(M)$ hat also nur Gültigkeit für den Aufbau der Geschlechtsdrüse, die also hiermit dem übrigen Körper als etwas völlig Gesondertes gegenübergestelit wird.

Wenn wir G. zunächst einmal in dieser Einteilung folgen, für die wir von unserem Standpunkte keine Berechtigung finden, so ergeben sich bei dem Versuche G.'s, seine Resultate auf zytologische Verhältnisse zurückzuführen, folgende Konsequenzen.

Verlegen wir den Geschlechtsbestimmer, wie üblich, in die Heterochromosomen und sehen das $O$ als heterozygot an (da in G.'s Zuchten zuerst nur gynandromorphe $q$ 우 auftraten), so ließe sich die Verteilung der "Geschlechtsbestimmer" im einfachsten Falle durch ein $Q \mathrm{X}$-Chromosom mit dem Faktor $F$ erklären ( $Q$ Ff $\sigma^{\lambda} \mathrm{ff}$ ). Dadurch, da $B$ das ㅇ aber Anlagen für $\delta$ und $q$ sekundäre Sexualcharaktere enthält, wird die Sache nur mit Hilfe von 2 ungleichen Chromosomen möglich. Sekundäre und primäre Sexualcharaktere sind korreliert, also in einem Chromosom vereinigt und wir würden demnach erhalten FGa (Weibchenbestimmer, y) und fgA (Männchenbestimmer, x). Das homozygote $\sigma^{t}$ enthielte dann 2 X-Chromosomen fgA. Nun kennen wir gerade unter den Insekten viele Formen, welche nicht $x$ - und y-Chromosomen, sondern nur eine Art von Heterochromosomen enthalten. In diesem Falle mu $B$ die Geschlechtsbestimmung neben dem Heterochromosom stets noch in einem andern morphologisch nicht differenzierten Chromosom vorhanden sein, resp. da diese stets paarig sind, in einem Paar von solchen. $\mathrm{Da}$ nun die sekundären und primären Sexualbestimmer korreliert sind, so müssen sie im gleichen Chromosom vereinigt, also der Faktor $\mathrm{F}$ auch homozygot im $\mathrm{O}$ vorhanden sein. G. kommt dabei endlich zu folgendem Schluß: Es gibt bei solchen Formen, wie die hier besprochenen, 4 Faktoren für die Geschlechtsbestimmung, $F G(q)$ und $\mathrm{MA}\left(\sigma^{\top}\right)$. Diese sind so verteilt, da $B$ das $\delta^{\top} 2$ X-Chromosomen mit MA enthält, das $O$ davon nur $I$. Beide Geschlechter enthalten außerdem je I Paar GF Chromosomen.

Mit Hilfe dieses Chromosomen und der Annahme, daß M und A über $\mathrm{G}$ und $\mathrm{F}$ epistatisch sind und $\mathrm{da} B$ die Potenz aller dieser Faktoren 
schwankt, wobei das epistatische Minimum gelegentlich unterschritten wird, kann G. tatsächlich alle seine Befunde darstellen. Er hat aber dabei die alte Auffassung der Erbfaktoren und ihre Verteilung in den Chromosomen völlig verschoben. Das Heterochromosom ist seiner Sonderstellung völlig entkleidet worden. Einmal reicht, wie wir eben sahen, die Annahme eines Heterochromosomes nicht aus, und in allen den Fällen, wo bei Insekten nur I Heterochromosom beobachtet ist, $\mathrm{muB}$ auf Grund dieser Kreuzungsergebnisse noch ein latentes Heterochromosomenpaar gefordert werden. Denn da aller Wahrscheinlichkeit nach bei allen Insektengruppen die somatischen Sexualcharaktere von denen der Keimdrüse unabhängig sind und der ganze somatische Erbkomplex sex-limited ist, so werden sich die Resultate der G.'schen Kreuzungen wohl für die Insekten verallgemeinern lassen.

Worin unterscheiden sich nun aber diese Heterochromosomen von den übrigen Chromosomen? $\mathrm{Da}$ sie Faktoren für „primäre und sekundäre Sexualcharaktere" per definitionem enthalten, so liegt in jedem Heterochromosom tatsächlich der Anlagekomplex für den ganzen Organismus. Es unterscheiden sich dann also die Heterochromosomen in nichts von den übrigen Chromosomen. Denn es genügt, wenn man sich auf den Boden der G.'schen Anschauung stellt, nicht etwa, in den geschlechtsbestimmenden Chromosomen einen generellen Faktor der Weibchen- oder Männchendetermination anzunehmen, sondern es muß jeder einzelne sexuell dimorphe Charakter im Heterochromosom vertreten sein, da die Mosaikvererbung ja auf einer verschiedenen Potenz der einzelnen Erbfaktoren beruht.

Es fällt also mit dieser Ableitung der Unterschied zwischen den normalen und den geschlechtsbestimmenden Chromosomen. Will man ihn aufrecht erhalten, so kommt man zu folgender Vorstellung. In den gewöhnlichen Chromosomen ist die Anlage der einzelnen Charaktere in „neutraler" Form gegeben. Die geschlechtsbestimmenden Chromosomen enthalten dann für jede dieser neutralen Anlagen einen Faktor, der sie in $\sigma^{\prime}$ oder $q$ determiniert. Diese Vorstellung scheint mir kaum durchführbar, wie sollten sich dann z. B. die Vererbungsverhältnisse gestalten, wenn ein Organ nur in einem Geschlecht sich verändert? Entweder muß dann der "neutrale“ Faktor mit verändert werden, oder der geschlechtsbestimmende Faktor muß, wenn er sich allein ändern soll, so beschaffen sein, daß neben ihm der neutrale völlig überflüssig erscheint.

Sind nach diesen Ausführungen qualitative Unterschiede, resp. das Vorhandensein spezieller geschlechtsbestimmender Chromosomen 
unwahrscheinlich geworden, so bliebe natürlich die Möglichkeit einer quantitativen Bestimmung zuzugeben. Wenn wir annehmen, daB bei gerader Zahl von Chromosomen (resp. Chromosomenpaaren) $\delta$ und $Q$ determinierte in gleicher $Z$ ahl vorhanden sind, so könnten natürlich Heterochromosomen quantitativ ausschlaggebend werden. $\mathrm{Ob}$ aber eine derartig grobe Vorstellung zulässig ist, erscheint mir sehr zweifelhaft.

M. E. deckt also gerade der bis ins äußerste durchgeführte Versuch GoLDSCHMIDT's, seine experimentellen Befunde mit den zytologischen Vorstellungen zu vereinigen, die inneren Widersprüche, welche in der Theorie der geschlechtsbestimmenden Chromosomen liegen, sehr klar auf. Un so angenehmer ist es nun für den, der die Tatsachen mehr vom physiologisch-chemischen Standpunkte betrachtet, da $B$ in den Ausführungen GoLDSCHMIDT's sich ein Punkt findet, der für physiologische Auffassung Ansatzpunkte bietet. Das ist der von ihm sehr stark ausgenutzte Potenzbegrif. Wenn er ihn auch noch in vererbungstheoretische Formeln kleidet und den Wechsel der "Potenz" als fluktuierende Variation der einzelnen Erbfaktoren auffaBt, so bleibt doch die Tatsache unbestreitbar, da $B$ mit ihrer Hilfe die Vererbungsträger sich aus starren Einheiten, die nur durch Anwesenheit oder Fehlen wirken können, in labilere, physiologisch zugänglichere Bildungen umwandeln. Ich vermute, $\mathrm{da} B$ der Ausbau des Potenzbegriffes ein Gebiet schaffen wird, auf dem sich die jetzt so scharf gegenüberstehenden Vererbungsmechaniker und Physiologen zu gemeinsamer Arbeit zusammenfinden können. 